\title{
Development of IME with Only One Switch for Physically Disabled Persons
}

\author{
Naruki Shirahama \\ Department of Creative Engineering, Information and Systems Engineering Course \\ National Institute of Technology, Kitakyushu College \\ 5-20-1, Shii, Kokuraminami-ku, Kitakyushu-shi, Fukuoka 802-0985, Japan \\ *Corresponding Author: naruki@kct.ac.jp
}

\begin{abstract}
It is essential for physically handicapped person to use auxiliary equipment for communication with others. We have developed a text input assistance application for mobile devices "RakuRaku IME" ("RakuRaku" means easy in English), the main user of this application is physically disabled person who cannot move his/her fingers and thumbs smoothly.

The effectiveness of the function has been confirmed by index defined by us. As the value of the index $C$ approaches 1 , it signifies that the input was performed without needless controls. A better interface might have a variety of elements, however to present the results of this study numerically, in this case we simply defined an index that emphasizes input speed.

We describe some features that we have implemented newly. One is the kana-to-kanji conversion function, which is implemented on the server. Settings of this app are often conducted by caregivers rather than the subject. Caregivers can manage the dictionary registration and priority of the word conversion on the server. The other is the setting function of character transition speed. We consider the character transition speed should be changeable.

We also reexamined text input processes. Character input is done through stepwise and hierarchical selection, however there are a few problems that impede improvement of input speed. To verify the effectiveness of this system, we have done several experiments with various conditions set. We examine these results and discuss future work.
\end{abstract}

Keywords: Assistive Technology, Human Interface, Android, Auto scan, Communication tools

\section{Introduction}

Smartphones and tablets have attracted a great deal of attention as equipment not only for non-handicapped person but also for disabled person. The main purpose of this research is, using the excellent features for communication with of these mobile devices, to improve the QOL of physically disabled person. The development of communication tools for the physically disabled person is also a significant research issue until now. One key issue is development and improvement of the text input interface. There are many constraints in the development of input interface for physically disabled person. This is because complex operations are difficult for him/her. We propose to use smartphones which have been popular in recent years. Our major goal is to develop a smartphone environment that is easy to use for physically handicapped people.

\section{Basic Overview of RakuRaku IME}

Here, we explain the input target character movement mechanism in detail. We explain the structure of Hiragana which is the basic character of Japanese. Figure 1 shows hiragana base characters and its transition to derived characters. Upper half of the figure shows hiragana base characters. The hiragana syllabary consists of 46 characters, 5 singular vowels $(\mathrm{a}, \mathrm{i}, \mathrm{u}, \mathrm{e}, \mathrm{o})$ conceived as a $5 \times 10$ grid. Basic hiragana characters can change to derived hiragana characters (lower half part of the figure)

To input one of the 50 Hiragana characters, first the character set columns from "a” (あ) to “wa” (わ) are displayed, as shown in figure 1 . 
For example, when the desired "ha" (は) character shown in figure 2 is shaded red, the button is pressed and the display changes to that shown in figure 3 (left), on the right, however, all characters in the "ha" (は) column are displayed, and selection moves periodically from one character to the next. When the desired character "fu" (ふ) is shaded red, the button is touched again, as shown in figure 3 (right) and the display change to that shown in figure 4 .

Selection moves between the normal character "ha" (は), the voiced sound "ba(ば)", and the semi-voiced sound “pa”(ぱ). For characters such as “a”(あ), selection moves between uppercase and lowercase characters. One character is input after the button is touched while the selected character is shaded red. And the next character in the text can be input.

\begin{tabular}{|c|c|c|c|c|c|c|c|c|c|c|}
\hline \multicolumn{4}{|c|}{ Base Characters } & \multicolumn{6}{|c|}{$\begin{array}{l}1^{\text {st }} \text { transition is the } \\
\text { first character of the first row }\end{array}$} & \multirow{7}{*}{$\begin{array}{l}2^{\text {nd }} \text { transition is } \\
\text { each character } \\
\text { on the selected } \\
\text { column }\end{array}$} \\
\hline わ & 5 & p & ま & は & $\begin{array}{l}\text { な } \\
\end{array}$ & た & さ & か & $\begin{array}{l}\text { あ } \\
\end{array}$ & \\
\hline Wa & $\mathrm{Ra}$ & $\mathrm{Ya}$ & $\mathrm{Ma}$ & $\mathrm{Ha}$ & $\mathrm{Na}$ & $\mathrm{Ta}$ & $\mathrm{Sa}$ & $\mathrm{Ka}$ & A & \\
\hline & $\eta$ & & み & ひ & に & ち & L & き & $w$ & \\
\hline & $\mathrm{Ri}$ & & $\mathrm{Mi}$ & $\mathrm{Hi}$ & $\mathrm{Ni}$ & $\mathrm{Ti}$ & $\mathrm{Si}$ & $\mathrm{Ki}$ & I & \\
\hline を & る & 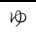 & む & 了 & ぬ & 0 & す & $<$ & $\overline{5}$ & \\
\hline Wo & $\mathrm{Ru}$ & $\mathrm{Yu}$ & $\mathrm{Mu}$ & $\mathrm{Hu}$ & $\mathrm{Nu}$ & $\mathrm{Tu}$ & $\mathrm{Su}$ & $\mathrm{Ku}$ & $\mathrm{U}$ & \\
\hline & れ & & め & $\sim$ & $\sqrt{2}$ & $\tau$ & 世 & け & 元 & \\
\hline & $\operatorname{Re}$ & & $\mathrm{Me}$ & $\mathrm{He}$ & $\mathrm{Ne}$ & $\mathrm{Te}$ & $\mathrm{Se}$ & $\mathrm{Ke}$ & E & \\
\hline ん & 3 & よ & क & ほ & क) & と & z & こ & 抒 & $\downarrow$ \\
\hline $\mathrm{Ng}$ & Ro & Yo & Mo & Ho & No & To & So & Ko & $\mathrm{O}$ & \\
\hline
\end{tabular}

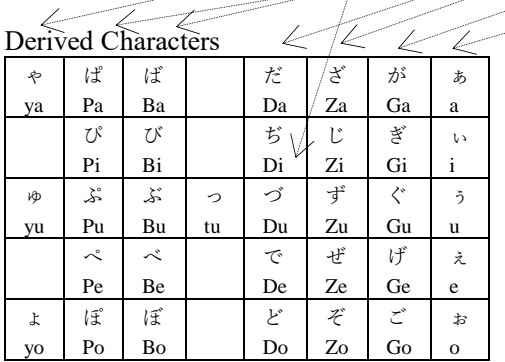

$3^{\text {rd }}$ transition

from the basic character to the corresponding derived character (if it exists)

Fig. 1 Transition of "Hiragana" Character

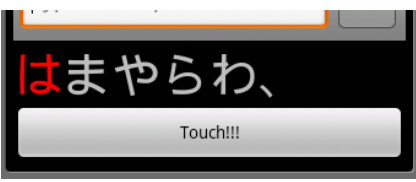

Fig. 2 The developed software keyboard.

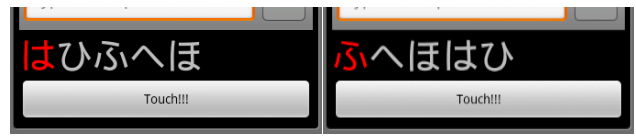

Fig. 3 Text movement of Step 2 in Fig. 2

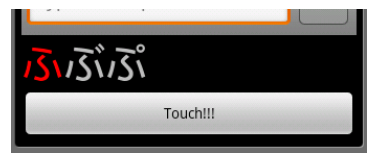

Fig. 4 Movement of Step 3 in Fig. 2

\section{Definition of Index for Ease of Input}

To define ease of input as the index, the equation designed by Shirahama, Sakuragi and $\mathrm{Kudou}^{(1,2)}$. As the value of $C$ approaches 1, it signifies that the input was performed without needless controls. A better interface might have a variety of elements, however, to present the results of this study numerically, in this case we simply defined an index that emphasizes input speed in equation (1). Figure 5 shows a diagram of the method for calculating the theoretical value and an example of the time to determine a single character expressed as a graph. Figure 6 shows the scenes using RakuRaku IME.

$$
C=\frac{T}{(\beta-\alpha) t+\alpha s}
$$

\section{$C$ Ease of use \\ $T$ Actual measured value \\ $\alpha \quad$ Number of required touches \\ $\beta \quad$ Number of required selection movements \\ $t \quad$ Selection movement interval \\ $s \quad$ Selection movement determination time}

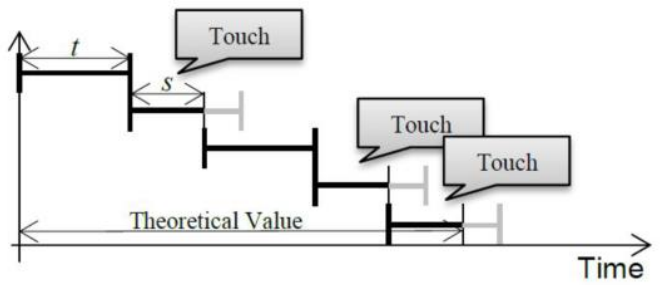

Fig. 5 Calculating theoretical value for determination time

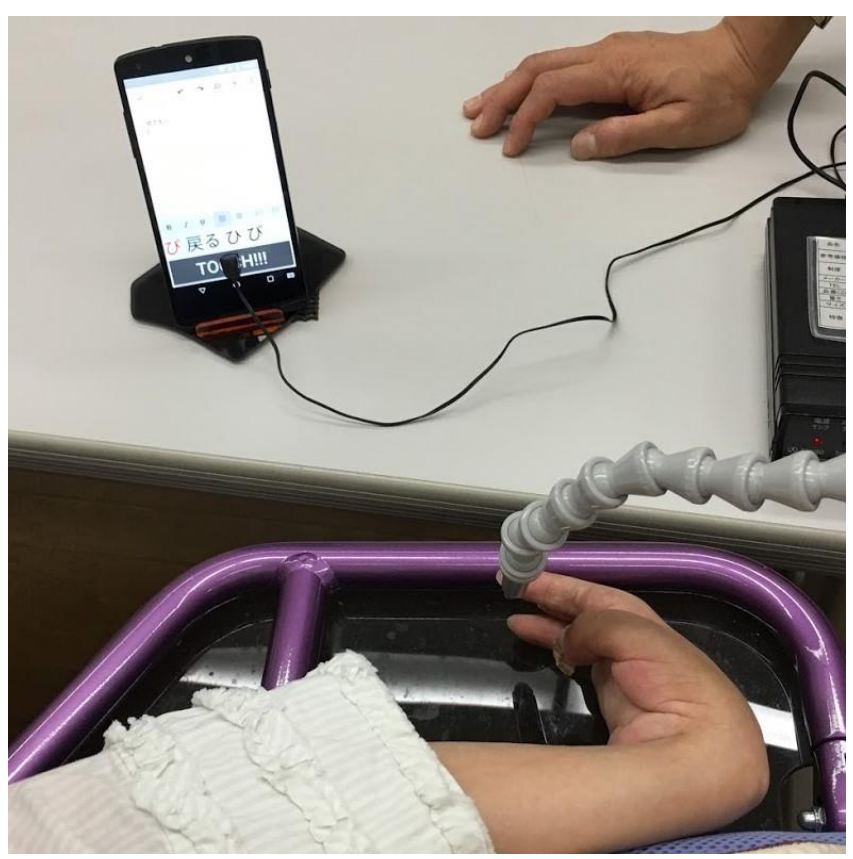

Fig. 6 Character input experiments to mobile devices 


\section{Kana-to-Kanji Conversion and Character Transition Speed Adjustment}

Caregivers can manage the dictionary registration and priority of the word conversion on the server. Character transition speed, which depends on the individual differences generally, also became changeable. Figure 7 is a usage history on the server. Figure 8 is a new setting screen for character transition speed, size of the beep sound and Enable switch for kana-kanji conversion.

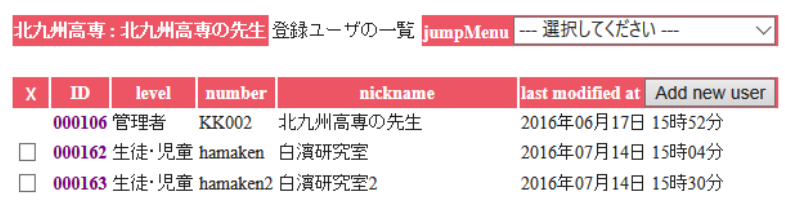

Fig. 7 User's conversion history on Server

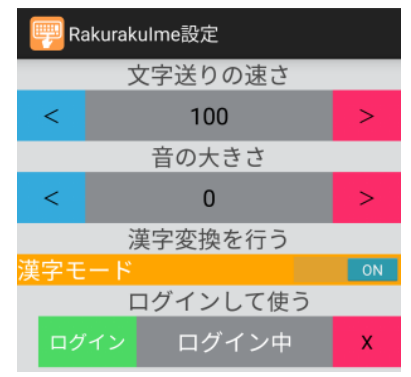

Fig. 8 New configuration item

\section{Improvement on IME character input}

To explore the IME's input improvement point, we visited the welfare equipment plaza Kitakyushu. We asked a collaborator, the patient of spinal muscular atrophy, to use the application to input the sentences. We explain the feedback items on the operation feeling obtained from the user this time.

\subsection{Reorganizing the IME input hierarchy}

We adjustment of input hierarchy of character. Table 1 and table 2

Tab. 2 shows first selection of character transition of conventional model and improved model, respectively. We could drastically reduce the number of initial transition candidate options from 17 to 4 .

Although the input hierarchy increases by one, it is still expected to shorten the speed time. We solved this problem by reducing the hierarchy on hold and confirmed conversion.
Tab. 1 First selection transition (conventional model)

\begin{tabular}{|c|c|c|c|c|c|c|c|c|c|c|c|c|c|c|c|c|}
\hline 1 & 2 & 3 & 4 & 5 & 6 & 7 & 8 & 9 & 10 & 11 & 12 & 13 & 14 & 15 & 16 & 17 \\
\hline A & KA & SA & TA & NA & HA & MA & YA & RA & WA & C1 & C2 & C3 & F1 & F2 & F3 & F4 \\
\hline あ & か & さ & た & な & は & ま & や & $ら$ & わ & & & & & & & \\
\hline
\end{tabular}

\begin{tabular}{|l|l||l|l|}
\hline C1 & comma, period, space & F1 & conversion function \\
\hline C2 & numerical character & F2 & confirm function \\
\hline C3 & symbolic-character & F3 & delete function \\
\hline
\end{tabular}

Tab. 2 First selection transition (improved model)

\begin{tabular}{|c|c|c|c|}
\hline 1 & 2 & 3 & 4 \\
\hline A-NA(あ-な) & HA-MA(は-わ & Character & Function \\
\hline
\end{tabular}

\section{あかさたな戻 TOUCH!!!}

conventional model

\section{あは点数記変 TOUCH!!!}

improved model
Fig. 9 Comparison of first selection transition string

\section{Experiment on Performance Comparison}

Experiments were conducted to compare the difference between the input performance of IME newly improved this time and the input performance of IME up to this year. Each IME used in the comparative experiment is shown in table 3. We prepared five kinds of applications, and measured the character input speed with each IME.

Tab. 3 Each IME used for comparison experiment

\begin{tabular}{|l|l|}
\hline IME 1 & Conventional IME \\
\hline IME 2 & Dictionary registration function \\
\hline IME 3 & Adjustment of input hierarchy of character \\
\hline IME 4 & Reduction of "Conversion" / "Hold" \\
\hline IME 5 & All the above elements (latest edition) \\
\hline
\end{tabular}

Experiments were conducted on 10 healthy volunteers, and the number of input errors and the number of input errors was recorded. Select transition interval is set to 1000 [ms] of the standard setting. Figure 10 shows the sentences used in the experiment and the number of characters and required transition times. We converted from "おひ" (o hi) to "お久し ぶりです" (o hi sa shi bu ri de su) in the experiment procedure using the dictionary registration function.

Table 4 shows the number of characters and the necessary number of transitions used for calculation of index $C$. Table 5 shows the average input time and the average number of input errors in the experiment, respectively. 
Tab. 4 Number of characters and the required number of transitions

\begin{tabular}{|c|c|c|}
\hline IME & $N$ & $T$ \\
\hline 1 & 17 & 230 \\
\hline 2 & 11 & 161 \\
\hline 3 & 17 & 207 \\
\hline 4 & 17 & 221 \\
\hline 5 & 11 & 132 \\
\hline
\end{tabular}

$N$ : Number of characters (Hiragana)

$T:$ Required number of transitions

$$
\text { お久しぶりです。お元気ですか? }
$$

(o hi sa shi bu ri de su, o ge n ki de su ka?)

(Long time no see. How are you?)

Fig. 10 Example sentence

Tab. 5 Average input times and numbers of input errors

\begin{tabular}{|c|r|r|}
\hline IME & Input time [s] & Input errors [n] \\
\hline 1 & 281.00 & 1.70 \\
\hline 2 & 210.40 & 1.20 \\
\hline 3 & 250.70 & 2.50 \\
\hline 4 & 253.40 & 1.50 \\
\hline 5 & 161.10 & 1.20 \\
\hline
\end{tabular}

As can be seen from table 5, these improvements have shortened the input time. All input times are shortened with each improvement factor, and latest version which includes every improvement are further shortened, therefore this result shows improvement of performance clearly. The average number of input errors is about 1 to 2 times, which has not changed much from the conventional number of times. We estimate that the reason why the number of mistakes in IME 3 is slightly higher is since the input procedure is significantly different from other IMEs.

Table 6 shows the usability index $C$ for each version. Because the time required for inputting the quickest becomes the closer to the measurement time, the value of index $C$ approaches from 0 to 1 , and the theoretical ease of use becomes greater.

Tab. 6 Values of index $C$ for each version

\begin{tabular}{|c|c|}
\hline Version & Index $C$ \\
\hline 1 & 0.826 \\
\hline 2 & 0.835 \\
\hline 3 & 0.742 \\
\hline 4 & 0.951 \\
\hline 5 & 0.892 \\
\hline
\end{tabular}

In the version that divides the first selection into two lines from "A line to a line" to "A line to a line," the index $C$ decreases slightly.

\section{Conclusions}

We added the functions to character input assistance application for physically handicapped persons, and obtained the following conclusions after verification and evaluation of its performance.

1. We improved conventional layout and improved operability.

2. We implemented kana-kanji conversion function which can be adjusted for each user on the server.

3. We succeeded in shortening the input time while maintaining the ease of inputting characters, because of improving the layout and adding functions.

Implementation of cancellation keys by long press operation, input position movement keys, a mode suitable for inputting numbers / alphabets, etc. is future tasks.

\section{Acknowledgment}

This research is one of the achievement of "Development of cloud environment to visualize educational effects of learning, and learning support applications", supported by the promotion project for utilizing teaching material and support equipment on learning by Ministry of Education, Culture, Sports, Science.

\section{References}

(1) S. Kudou and N. Shirahama : "Improvement of Operability in Input Assistance Application for Mobile Devices for Physically Disabled", 4th International Symposium on Technology for Sustainability, 2014.

(2) N. Shirahama, Y. Sakuragi, S. Watanabe, N. Nakaya, Y. Mori and K. Miyamoto : "Development of Input Assistance Application for Mobile Devices for Physically Disabled, 15th IEEE/ACIS International Conference on Software Engineering, Artificial Intelligence, Networking and Parallel/Distributed Computing, pp. 411-416, 2014. 